\title{
LOS CONTENIDOS TEMÁTICOS Y SOCIOCULTURALES DE LOS MANUALES DE F.L.E. PARA PRIMARIA
}

\author{
Ma José Bonachía Caballero \\ Universidad de La Rioja
}

\section{RESUMEN}

El aspecto sociocultural de la lengua extranjera adquiere nueva relevancia en la enseñanza-aprendizaje en las nuevas leyes educativas como consecuencia de la publicación del MERC en 2001.

Teniendo en cuenta los nuevos objetivos, este artículo pretende analizar los contenidos temáticos y socioculturales de algunos manuales de F.L.E. para Primaria, de manera general y, de tres documentos en particular: Le Poisson d'avril, Menu cantine y La famille de Théo.

La conclusión es que el material sigue siendo válido teniendo en cuenta que en esta etapa educativa la labor del profesor es fundamental para actualizar, matizar e integrar los contenidos en el aula.

PALABRAS CLAVE: Enseñanza de lengua extranjera, aspecto sociocultural de la lengua, manuales y textos de Primaria.

\section{ABSTRACT}

As a consequence of the publication of the MER in 2001, the sociocultural aspect of foreign language learning and teaching takes added significance in new education laws. Bearing in mind the new objectives, this paper attemps to analyse the thematic and socio cultural contents of a number of F.F.L. texts for primary education, and specially three of them: Le Poisson d'avril, Menu cantine and La Famille de Théo.

The evaluation of these teaching materials has been done taking into account that the that in this educational period, the word of the teacher is essential in order to update, specify, and integrate contents in the classroom.

KEYWORDS: foreing language teaching, socio cultural aspect of language, course books. 


\section{1.- Introducción: El Marco Europeo Común de Referencia y los objetivos sociocul- turales en Primaria}

El Marco Europeo Común de Referencia para las Lenguas (en adelante, MECRL), (Conseil de l'Europe, 2001) y, como consecuencia de éste, la nueva ley de educación vigente, conceden gran importancia al aspecto sociocultural de la Lengua Extranjera (en adelante, LE) en el proceso de enseñanza-aprendizaje desde las primeras etapas educativas. Teniendo en cuenta este presupuesto, nos proponemos hacer una reflexión sobre los contenidos temáticos y culturales de algunos de los manuales de Francés Lengua Extranjera (en adelante, FLE) más utilizados para Primaria en general, y de tres documentos en particular, para medir el grado de adecuación de éstos a los nuevos objetivos educativos en los que la dimensión cultural ocupa un lugar preponderante.

Aunque todas las competencias humanas contribuyen, de una u otra forma, a la capacidad comunicativa del usuario, y se pueden considerar aspectos de la competencia comunicativa, conviene distinguir entre las competencias generales, menos relacionadas con la lengua, y las competencias lingüísticas propiamente dichas (MCERL, 2002).

El conocimiento sociocultural que forma parte de las competencias generales es definido como "el conocimiento de la sociedad y de la cultura de la comunidad o comunidades en las que se habla el idioma". Sin embargo, el concepto de cultura es muy amplio, y tanto de éste como de sus derivados: multiculturalidad, pluriculturalidad e interculturalidad (Trujillo, 2005) se han dado ya diferentes definiciones, basadas en la sociología ${ }^{[1]}$, la antropología y la sociolingüística ${ }^{[2]}$.

En cualquier caso, aprender una lengua extranjera supone aprender un nuevo código de comunicación, diferente al de la comunidad de origen, y el alumno tiene que saber que comprender ese código implica adquirir una visión diferente del mundo. Además, la comunicación como práctica social no se reduce a la comunicación lingüística, pues intervienen otros factores extralingüísticos y no verbales, como son: la manera de vestirse, los modales, los gestos, el tono de voz, la forma de sentarse, la forma de estar, de mirar, etc. Todos estos factores están codificados por la cultura en formas de cortesía, normas de educación, reglas estéticas y morales y, además, varían dentro de una misma cultura ya que son distintos según el grupo social en el que se está inmerso. Por ello, el lenguaje debe ser tratado en un contexto social, pues la relación entre lenguaje y sociedad es capital para la enseñanza de la LE, en la medida en que la finalidad del aprendizaje de una LE tiene que ver con "establecer contacto" y "comunicación" con otro grupo cultural. Por tanto, sociedad y cultura son conceptos indisociables que representan a las personas con las que los alumnos de LE van a comunicarse e interactuar.

Del mismo modo, la nueva ley educativa ${ }^{[3]}$ dice que, el eje de la LE en Primaria lo constituyen los procedimientos dirigidos a la consecución de una competencia

[1] La sociología y la antropología proporcionan instrumentos para el estudio sistemático de las sociedades y las culturas del que se derivan los contextos necesarios para un estudio del lenguaje y sus respectivas aplicaciones al ámbito de la LE.

[2] La sociolingüística proporciona una serie de conceptos e información sistemática para un estudio del lenguaje en un medio social, cultural e interpersonal.

[3] Ley Orgánica 2/2006, de 3 de mayo, de Educación. BOE nº106 de 4/5/2006. 
comunicativa efectiva oral y escrita, en contextos sociales significativos. Asimismo que el aprendizaje de una LE ha de contribuir al desarrollo de actitudes positivas y receptivas hacia otras lenguas y culturas, $y$, al mismo tiempo ayudar a comprender $y$ valorar la lengua o lenguas propias.

Los contenidos se agrupan en cuatro bloques que ordenan los elementos de análisis de una realidad compleja, en relación a cuatro centros de atención con características y necesidades específicas en el proceso de enseñanza y aprendizaje. Los tres primeros incluyen las destrezas lingüísticas y comunicativas: el lenguaje oral; el lenguaje escrito; y los elementos constitutivos del sistema lingüístico, su funcionamiento y relaciones. El cuarto bloque se refiere específicamente a la dimensión social, rasgos y particularidades de los países en los que se habla la LE.

La importancia de los aspectos socioculturales en la enseñanza de la LE en Primaria queda reflejada, sobre todo, en los siguientes objetivos: ${ }^{[4]}$

- Actitud receptiva hacia las personas que hablan otra lengua y tienen una cultura diferente a la propia.

- Conocimiento de algunas similitudes y diferencias en las costumbres cotidianas y usos de las formas básicas de relación social entre los países donde se habla la $L E$ y el nuestro.

- Valoración de la LE o de otras lenguas como medio para comunicarse y relacionarse con compañeros de otros países, como posibilidad de acceso a informaciones nuevas y como instrumento para conocer culturas y modos de vivir diferentes y enriquecedores.

- Interés por establecer contactos y comunicarse con hablantes de la LE o de otras lenguas a través de los medios que nos proporcionan las tecnologías de la comunicación.

Frente a la cultura más formal que incluiría la historia, las artes y los grandes logros de una comunidad, estos objetivos presentan una perspectiva de la cultura en aspectos no formales: vida cotidiana, valores, creencias, costumbres... y una intencionalidad formativa que implica la capacidad de reflexionar sobre la propia cultura y la extranjera, así como de las habilidades necesarias para relacionarse con el mundo del otro.

Por otro lado, el conocimiento y la comprensión de las similitudes y diferencias existentes entre el propio universo cultural y el de la comunidad donde se habla la LE desarrolla en el alumno una conciencia intercultural que incluye la idea de una diversidad social de ambos universos. Además, la formación escolar y la didáctica de la lengua contribuyen al desarrollo del conocimiento sociocultural, sobre todo en la medida en que se incorporen los contenidos de las áreas curriculares a la enseñanza de la lengua.

El MCERL adopta un enfoque centrado en la acción, en la medida en que considera a los alumnos que aprenden una lengua, principalmente, como agentes sociales, es decir, miembros de una sociedad que tiene tareas que Ilevar a cabo en una serie determinada de circunstancias, en un entorno específico y dentro de un campo

[4] RD 1513/2006, de 7 de diciembre, por el que se establecen las enseñanzas mínimas de la Educación Primaria. BOE nº 293 8/12/2006. 
de acción concreto. Y es que los alumnos necesitan tomar parte activa en las interpretaciones del mundo, encontrando fenómenos que expresen parte del significado compartido en la LE que puedan contrastarse y compararse con la lengua materna. Por ello, en la etapa de Primaria, la iniciación a la cultura de la LE se puede hacer partiendo de los conocimientos y representaciones que tienen los alumnos del país extranjero y de sus gentes, e intentando objetivar desde el primer momento la relación entre las dos culturas.

Los medios tecnológicos, al alcance de una gran mayoría de alumnos, pueden suponer un apoyo importante para este propósito en el proceso de enseñanza-aprendizaje de la LE. El uso del ordenador con programas de texto, las enormes posibilidades de recibir información a través de Internet, la comunicación a través del correo electrónico, los foros de discusión o la comunicación de clase a clase, posibilitan los contactos entre aprendices de distintos países y culturas mediante la indagación y la interacción.

Sin perjuicio de lo expuesto, en esta etapa escolar es fundamental el papel del profesor para mediar y aportar un uso humanístico de las nuevas tecnologías, y evitar así una utilización pasiva y mecánica de las mismas.

\section{2.- Contenidos temáticos y culturales de los manuales de Primaria}

Antes de analizar y evaluar un material para la enseñanza del componente cultural algunos autores proponen un modelo de análisis en torno a cinco apartados como son el marco de producción, la información y tratamiento de la información, la integración de lengua y cultura, los aspectos socioculturales de la lengua meta y por último la programación de la cultura meta (Areizaga, 2002). Por su parte, otros autores hacen una clara distinción entre los códigos icónico y lingüístico y articulan el análisis sobre dos grandes ejes: las imágenes y los textos (Paricio, 2005).

De todos los aspectos citados, nos interesa destacar especialmente el de la integración de lengua y cultura, pues en efecto, el desarrollo de la competencia comunicativa lleva implícito el trabajo de los aspectos socioculturales como parte central del aprendizaje lingüístico, dentro del contexto de las actividades del aula, independientemente de que el trabajo se realice con un texto o con una imagen.

Esta reflexión se basa en el análisis de los contenidos temáticos y culturales de algunos de los manuales de FLE para Primaria más utilizados como Farandole 1 y 2 (Le Hellaye, 1992-1993), Kangourou (Paccagnino-Poletti, 1992), Trampoline (MeyerDreux, 1991), La Marelle (Candela et al. 1997), Carambole, 1 y 2 (Juri-Guiard 2003) o Mikado (Palomino, 2003).

En dicha reflexión se parte de una visión general de los temas, teniendo en cuenta que el libro de texto es una representación de la forma en la que el autor y el editor conciben la lengua, la cultura y el aprendizaje, así como la forma en que construyen un mundo integrado de realidad extranjera para propósitos de instrucción (Kramsch, 1988).

Así, en los manuales citados, encontramos propuestas variadas para trabajar la lengua oral, con diferentes tipos de discursos como las comptines o canciones, pequeños relatos, diálogos con registros de la lengua oral, además de vocabulario y estructuras lingüísticas.

En cuanto a la lengua escrita, los tipos de textos y soportes que aparecen son familiares para los niños, como pueden ser: cómics, diálogos, consignas de ejercicios, 
reglas de juegos, fichas de bricolaje, recetas de cocina, textos poéticos o culturales, anuncios publicitarios, cartas, tarjetas, notas, etc.. Todos ellos se refieren a los temas dominantes que interesan a los niños entre ocho y doce años pues están relacionados directamente con su vida y sus actividades cotidianas, siendo, por esta razón, temas recurrentes.

Los contenidos temáticos más relevantes de los manuales podemos agruparlos en torno a tres grandes apartados, como son: la escuela, la casa y las actividades de ocio y tiempo libre. El primero de ellos constituye un tema nuclear y las actividades planteadas presentan la jornada ordinaria de un escolar, el espacio de la clase, la correspondencia tradicional y la electrónica, (en los manuales más recientes, prácticamente ha sustituido a la primera) los juegos y todo lo que constituye el universo escolar.

Las actividades siguen el ritmo del calendario, comenzando en el mes de septiembre con el inicio del curso que marca la vuelta al colegio. La vida escolar está regida por los distintos períodos vacacionales, siguiendo las estaciones y las diferentes fiestas tradicionales que tienen lugar a lo largo del año y en las que los niños son los protagonistas: Navidad, Carnaval, Poisson d'Avril, los cumpleaños, las fiestas del colegio, las fiestas locales, etc., que van a marcar muchas de las actividades en el aula de LE.

Otro gran apartado temático es el que se refiere a la familia, las profesiones, los valores y costumbres familiares en cuanto a la comida, el espacio y el equipamiento de la casa, la ropa, los animales domésticos, etc.

El tercer bloque está dedicado fundamentalmente al tiempo de ocio de los niños en diferentes ámbitos: los jardines, la piscina, el zoo, el circo, etc. así como la práctica deportiva cotidiana. Incluimos también es este apartado las vacaciones y el tiempo meteorológico e igualmente al conocimiento de ciudades y paisajes franceses, los parques temáticos, los países de la comunidad europea y los medios de transporte.

Estos temas están presentados en los manuales a partir de actividades lúdicas, muy variadas y atractivas para los niños, porque el juego y la dimensión lúdica expresiva, como una forma de dramatización, puede considerarse como una respuesta total al proceso de aprendizaje lingüístico que incluye lo verbal y no verbal. Desde la psicología, el juego constituye la actividad nuclear en el proceso de aprendizaje lingüístico (Vigotsky, 1991), y concentra en sí mismo todos los procesos del desarroIlo madurativo del niño.

Estas actividades se refieren a la fabricación de pequeños objetos necesarios para las fiestas señaladas, como una guirlanda de Navidad, un muñeco de nieve, un sombrero de carnaval, una marioneta, etc. a la realización de sencillas recetas de cocina, de juegos diversos, a la recitación o interpretación de comptines, canciones tradicionales o bien, canciones infantiles modernas y también a la dramatización de cuentos tradicionales adaptados u otros tipos de cuentos.

\section{3.- Documentos analizados desde el punto de vista sociocultural}

\section{a) Le Poisson d'avril}

El tema de las páginas dedicadas al "premier avril", [Farandole, 2, 50-53] (Le Hellaye, 1992-1993) responde a una tradición como hecho cultural común, que deviene de la supervivencia de otras manifestaciones culturales que se insertan en la historia del país. Es una costumbre que se manifiesta en la escuela y en otros ámbi- 
tos sociales, especialmente en los medios de comunicación. Ese día los niños recortan peces de papel que cuelgan en la espalda de los compañeros con la intención de gastarse bromas y reírse de los demás.

La noticia que se pasan los escolares de que van cerrar las escuelas durante una semana, la falsa ficha de bricolaje según la cual alguien resultaría empapado de agua, el anuncio en la televisión de que van a desmontar la Tour Eiffel o que el partido de fútbol de la Copa del mundo tendrá lugar en el parque del castillo de Versalles, son algunas de las bromas que aparecen en los textos de referencia.

Sobre el Poisson de avril, se cuenta que hasta 1564 en Francia el año comenzaba el 1 de abril, pero el rey Carlos IX decidió modificar el calendario y a partir de esa fecha el año comenzaría el 1 de Enero, día en el que todos seguirían deseándose un feliz año y haciéndose regalos. Sin embargo, cuando llegó el 1 de abril, algunos farsantes tuvieron la idea de continuar con los regalos como se hacía antes. Pero puesto que eran farsantes, los regalos fueron falsos obsequios, ideados sólo para hacer reír.

Como en esta época del año en Francia está prohibido pescar por ser período de reproducción de los peces, algunos tuvieron la idea de hacer bromas a los pescadores echando arenques al río mientras gritaban: ¡Pez de abril! y de esta forma se mantuvo la costumbre que ha pervivido hasta la actualidad. Aunque hoy en día ya no se lanzan arenques al agua todos, pequeños y mayores, han adoptado el hábito de las chanzas, si bien es cierto que los niños son los que más disfrutan cuando preparan los motivos de broma.

En otros países se sigue también esta tradición de engañar y hacer bromas el 1 de abril, como por ejemplo en el Reino Unido y en Estados Unidos, donde se celebra el April's Fool's Day o "día de los tontos de abril"; en Italia se llama Pesce d'aprile y en Alemania simplemente 1 April, aunque cada país busca el origen en su propio acervo cultural.

Lo mismo ocurre en España y en Hispanoamérica, donde las bromas (inocentadas) se celebran el 28 de diciembre, día de los Santos Inocentes. Ese día se conmemora el hecho bíblico de la matanza de todos los niños menores de dos años nacidos en Belén, ordenada por el rey Herodes con el fin de deshacerse del recién nacido Mesías. Las bromas que se hacen hoy en día podrían deberse al recuerdo del modo de ser inocente de aquellos bebés que no tuvieron tiempo de jugar ni reír.

Por supuesto, en torno al Poisson d'avril se pueden desarrollar multitud de actividades pedagógicas (confeccionar y enviar tarjetas coloreadas o con textos divertidos, imaginar recetas de cocina, juego de las diferencias entre dos dibujos similares, canciones, pequeñas lecturas sobre distintos tipos de peces, etc.) Pero además, con la explicación del origen de la tradición, el profesor puede invitar a los niños a que cuenten a su vez, qué tipo de bromas hacen para celebrar ese día en la cultura de sus países de origen, siempre y cuando exista la costumbre.

\section{b) Menú cantine (11-17 mars)}

El segundo documento [La Marelle, p. 10] (Candela et al, 1997), con una clara función ilustrativa del vocabulario presentado, es un texto incluido en una página dedicada a la lectura, muy sobrio en su presentación pero que aporta mucha información desde diferentes perspectivas como pueden ser la lingüística, la social, la educacional y la cultural. 
Desde el punto de vista lingüístico, contiene una lista de vocabulario específico referido a los alimentos y a la comida de un menú escolar (radis, saucisson, tomates, carottes rapées, pâté/salade, saucisse/purée, rôti/haricots, viande/nouilles, poulet/frites, poisson/riz, yaourt, fromage, crème, glace, orange, banane, pomme), vocabulario que puede relacionarse y ampliarse fácilmente con el tema genérico de la comida.

Por su parte, el aspecto social comporta una estructura con respecto a la comida que se manifiesta en el hecho de trasladar la comensalidad en familia al contexto escolar: en el colegio los alumnos comen juntos y acompañados de los profesores, un menú planificado en su composición y variado en su presentación, lo que da un sentido social al hecho de comer y alimentarse.

Desde el punto de vista educacional, el texto permite abordar los hábitos de alimentación, la actitud ante los alimentos, la corrección del comportamiento en la mesa y, en fin, se puede hacer una didáctica de la nutrición.

El aspecto cultural del texto es la denominada "cocina francesa", emblemática entre las categorías gastronómicas mundiales, pues por un lado presenta un "menú tipo" caracterizado por una variedad de alimentos de origen y, por otro lado, permite fácilmente la comparación con el menú habitual del alumno. El documento puede servir de punto de partida para que el profesor explique otros aspectos culturales como el hecho de que las comidas familiares festivas aún conservan la tradición de comer cinco platos: una entrada, un plato principal (carne o pescado) acompañado de legumbres o verduras, ensalada, queso y postre. Puede además presentar otros platos y alimentos típicos de diferentes regiones francesas (la choucroute, la fondue savoyarde, la salade niçoise, le gâteau basque o la tarte tatin también aparecen en los manuales), así como otros tipos de comida rápida ampliamente extendidos, sobre todo entre los jóvenes, servidos en establecimientos como croisanterias, bocaterías, pizzerías, hamburgueserías, etc.

En este sentido, los franceses, al igual que ocurre en otros países desarrollados, se han abierto a las diferentes cocinas del mundo y en los supermercados se pueden encontrar platos y productos alimenticios de muchas nacionalidades (italiana, española, china, india, thaï, árabe, tropical, etc.) Por estas razones, el menú analizado, si bien forma parte de la identidad francesa, sobrepasa el marco de su gastronomía ya que permite incluir otras, como hemos indicado.

\section{c) La famille de Théo}

El tercer documento [Carambole 1] (Juri, L. Guiard, L., 2003), es una página ilustrada que alude a otro tema recurrente como es el significado de la idea de la familia y de la trascendencia individual y social como núcleo cultural.

En el dibujo se trata sin duda de la representación de un día especial, en el que todos los miembros de la familia están reunidos alrededor de una mesa compartiendo una tarta que simboliza una celebración o encuentro festivo. La escena recogida en una fotografía de gran tamaño, enmarcada como si de un cuadro se tratara, sirve a Theo para guardar en el recuerdo una reunión familiar excepcional de un día de fiesta. Por esta razón, si convenimos que se trata de una ocasión especial y no de un día cualquiera, el modelo de familia presentado tampoco es el habitual sino un prototipo. 
Como en el documento anterior, la imagen sirve para ilustrar los contenidos lingüísticos presentados en torno a cada uno de los integrantes de la familia aunque, lógicamente, hay mucha más información añadida como ocurre con cualquier imagen. La colocación de los personajes, su aspecto físico, su color de piel y de cabeIlo, su edad, su estado de salud, su vestimenta, sus gestos y actitudes, etc. así como el fondo en el que se enmarca la fotografía, el decorado, el mobiliario, los elementos de la mesa, por citar algunos aspectos, aportan una serie de indicios socioculturales que también podrían ser objeto de análisis, pero en los que no vamos a entrar.

El prototipo de familia es el de la familia tradicional extensa, formada por los padres, los abuelos y varios hijos, perteneciente a una clase social media, a juzgar por las respectivas profesiones de padres y abuelos (pintor, enfermera, profesor y periodista), el tipo de casa en la que viven y otros datos derivados de la observación de la imagen. La inclusión de Foufou, el perro, en el grupo familiar de Théo forma parte de un hecho habitual de la vida familiar francesa, donde las mascotas están totalmente integradas en un mayor porcentaje que en nuestro país ${ }^{[5]}$.

Sin embargo, la evolución social ${ }^{[6]}$ ha variado hacia otros modelos familiares que coexisten con el tradicional, siendo varias las posibilidades tanto si las consideramos desde el punto de vista de los vínculos de la pareja: familia natural, reconstruida, monoparental, como de la filiación de los propios hijos: biológicos, medio hermanos, hijos adoptados, en acogimiento, hijos únicos, etc.

Será tarea del profesor abordar otros modelos y situaciones de familia en el momento y la forma que estime oportuno, seguramente, con la posibilidad de hacerlo a partir de las experiencias de sus propios alumnos.

\section{4.- Conclusiones}

La conclusión que se aporta es que, tanto en los documentos de los manuales de Primaria analizados, como en los de otras etapas escolares, aparecen tópicos y estereotipos -inevitables- cuando se trata de presentar verdades o caracterizaciones generales de un país.

Lo que ocurre con los estereotipos es que, por un lado, la lengua, las personas y los países están caracterizados intrínsecamente, y por otro lado, la sociedad y sus representaciones evolucionan rápidamente. Por ello, es conveniente estar atento a la diversidad de las prácticas individuales y de las formas culturales del grupo-clase, tratando de ver en el otro los puntos comunes, como factor de acercamiento entre ambos grupos.

En esta etapa educativa es posible afirmar que los contenidos de los manuales siguen siendo válidos en la medida que tienen la función de servir de apoyo en la dimensión instrumental del aprendizaje, siempre que vayan acompañados de una

[5] Vid. (2006) Dossier Les animaux et nous, une nouvelle cohabitation. Le Français dans le monde, 348

[6] Según revelan los datos del Instituto Nacional de Estadística y del Consejo General del Poder Judicial, la familia española ha sufrido fuertes cambios, especialmente en la última década. El aumento de parejas de hecho, de bebés nacidos fuera del matrimonio y de hogares monoparentales, son tres de los factores que marcan el cambio. El País, 19 de Junio de 2005. 
aportación del profesor, para dar una mayor variedad y flexibilidad, actualizar y matizar, si es necesaria, la presentación de los contenidos en el aula.

En este sentido, la utilización paralela de documentos auténticos, debidamente seleccionados, puede contrarrestar el carácter monolítico y universal del material didáctico de los manuales en general e igualmente lo hará, la utilización de las nuevas tecnologías, tal como apuntábamos al principio.

Además, la clase de LE en el medio escolar no puede circunscribirse en exclusiva a los temas planteados en los manuales, sino que éstos deben integrarse en otras áreas del currículo y con los contenidos transversales, ayudando a los alumnos a reconstruir una cultura articulada, en lugar de fragmentada (Vez, 2001). Y así por ejemplo, si abordamos el tema de la alimentación, podemos enlazarlo con la educación para la salud y la adquisición de buenos hábitos alimenticios, o con los problemas actuales derivados de su inobservancia como son la obesidad, la anorexia o la bulimia.

Es una tarea en la que los profesores de LE debemos implicarnos a fondo, aportando nuestra visión del mundo y nuestro compromiso con el resto de profesores, formando alumnos competentes en LE es decir, desarrollando su capacidad de producir e interpretar lo que se dice de acuerdo con un contexto y adaptar los usos de la lengua a las diferentes situaciones de comunicación.

Así, tendremos que orientar los materiales contenidos en los manuales existentes y los nuevos que vayan apareciendo, ${ }^{[7]}$ hacia la consecución de la competencia intercultural propuesta en el marco de referencia común ya que la sociedad en la que vivimos está formada por ciudadanos de diferentes etnias, nacionalidades, culturas, lenguas y religiones.

Dicho esto, ya hemos apuntado que en el aprendizaje de una lengua extranjera la competencia comunicativa no se limita a la competencia lingüística, que el esfuerzo principal debe recaer en el conocimiento práctico de las reglas sociales y de las manifestaciones culturales que tienen en cuenta las relaciones interpersonales y el contexto, muy difíciles de adquirir en una situación de aprendizaje guiado. Por estas razones, deberían propiciarse al máximo los intercambios escolares y las estancias lingüísticas de los niños en el país de la LE, de igual modo que para los profesores de Primaria. ${ }^{[8]}$

\section{Bibliografía}

Areizaga, E. (2002) El componente cultural en la enseñanza de lenguas: elementos para el análisis y la evaluación del material didáctico. Cultura y Educación, 14 (2) 161-179.

[7] Una de las finalidades del MERC es precisamente la de servir de guía a los autores de libros de texto.

[8] Según el informe del Proyecto Trafic, que tiene como objetivo primordial fomentar el desarrollo de cursos para profesores de idiomas de Primaria en Europa, la integración de los aspectos lingüístico, profesional, intercultural y personal de estos cursos han sido identificados por el equipo del proyecto como un principio clave de "mejores prácticas". 
Auger, N. (2003) Manuels et stéréotypes. Le Français dans le monde, nº 326.

Barret, P., Gurgand, J.N., Tiévant, Cl. (1980) Almanach de la mémoire et des coutumes. Paris: Hachette.

Candela, P., et al, (1997), La marelle, Français langue vivante, Barcelona: Coiné.

Conseil de l'Europe (2001). Cadre européen commun de référence pour les langues. Apprendre. Enseigner. Evaluer, Paris, Didier/Conseil de l'Europe.

Cuq, J.P.; Gruca, I. (2003) Cours de didactique du français langue étrangère et seconde. Grenoble: Presses Universitaires de Grenoble.

Juri, L. Guiard, L. (2003) Carambole, Méthode de français, 1 y 2, Madrid: S.M.

Kramsch, C. (1988). The cultural Discourse of Foreing Language Textbooks. En: A.J. Singerman. Toward a New Integration of Language and Culture, pp 47-62. Middlebury. VT: Northeast Conference on the Teaching of Foreing Languages.

Le Hellaye, C. Barzotti, D. (1992-1993) Farandole 1 y 2, Méthode de français, París: Hatier: Didier.

Marco Común Europeo de referencia para las lenguas (MCERL): aprendizaje, enseñanza, evaluación (2002). Ministerio de Educación, Cultura y Deporte. Madrid: MECD y Grupo Anaya, p. 99.

Meyer-Dreux, S., Garabédian, M., Lerasle, M., (1991) Trampoline, Méthode de français. Paris: Clé International.

Palomino, M. (2003). Mikado, Madrid: Oxford Educación.

Paccagnino, C., Poletti, M.L. (1992), Kangourou, Méthode de français pour les enfants, Paris: Hachette.

Paricio Tato, M.S. (2005) La dimensión cultural en los libros de texto de lenguas extranjeras: pautas para su análisis. Glosas Didácticas, 15, 133-144.

Poirier, Cl. (avril 1970). Le mot de la semaine. Poisson d'avril. Chronique linguistique du TLFQ.1, 1. Extraído el 1 de abril, 2007 del sitio web de l'Université Laval de Québec (Canadá). http: www.tlfq.ulaval.ca/cours/chronique/chronique1.htm

Rico Martín, A. (2004) De la competencia intercultural en la adquisición de una segunda lengua o lengua extranjera: conceptos, metodología y revisión de métodos. Porta Linguarum, 3, 79-94.

Transnational Framework for Inservice Courses (Trafic), (2004). Aprender a enseñar otro idioma en la escuela primaria. CILT.

Trujillo Sáez, F. (2005) En torno a la interculturalidad: reflexiones sobre cultura y comunicación para la didáctica de la lengua, Porta Linguarum, 4, 23-39.

Trujillo Sáez, F. (2006) La cultura en la enseñanza de idiomas: de la teoría a la práctica a través del PEL. Articles de Didáctica de la Llengua i de la Literatura, 39, 4955.

Vez Jeremías, J. M. (2001) Formación en Didáctica de las Lenguas Extranjeras, Rosario: Homo Sapiens.

Vigotsky, L. (1991), El papel del juego en el desarrollo del niño, en : El desarrollo de los procesos psicológicos superiores. Barcelona: Crítica.

http://www.edufle.net/breve67.html. Activités pédagogiques sur le thème du "poisson d'avril" (2004, abril 6) Origine et tradition. Extraído el 1 abril, 2007. 